\title{
BEST CONSTANT IN ZYGMUND'S INEQUALITY AND RELATED ESTIMATES FOR ORTHOGONAL HARMONIC FUNCTIONS AND MARTINGALES
}

\author{
ADAM OSȨKOWSKI
}

\begin{abstract}
For any $K>2 / \pi$ we determine the optimal constant $L(K)$ for which the following holds. If $u, \tilde{u}$ are conjugate harmonic functions on the unit disc with $\tilde{u}(0)=0$, then$$
\int_{-\pi}^{\pi}\left|\tilde{u}\left(e^{i \phi}\right)\right| \frac{\mathrm{d} \phi}{2 \pi} \leq K \int_{-\pi}^{\pi}\left|u\left(e^{i \phi}\right)\right| \log ^{+}\left|u\left(e^{i \phi}\right)\right| \frac{\mathrm{d} \phi}{2 \pi}+L(K) .
$$

We also establish a related estimate for orthogonal harmonic functions given on Euclidean domains as well as an extension concerning orthogonal martingales under differential subordination.
\end{abstract}

\section{Introduction}

The motivation for the results obtained in this paper comes from a classical problem of comparing the sizes of a harmonic function and its conjugate. To be more specific, let $p \geq 1$ and suppose that $u$ is a real-valued harmonic function on the open unit disc $\mathbb{D} \subset \mathbb{C}$, satisfying

$$
\|u\|_{p}=\sup _{0<r<1}\left(\int_{-\pi}^{\pi}\left|u\left(r e^{i \phi}\right)\right|^{p} \frac{\mathrm{d} \phi}{2 \pi}\right)^{1 / p}<\infty .
$$

Let $\tilde{u}$ denote the harmonic conjugate of $u$, normalized so that $\tilde{u}(0)=0$. A classical theorem of M. Riesz (see [13] and [14]) states that if $1<p<\infty$, then for some $C_{p}$ depending only on $p$ we have

$$
\|\tilde{u}\|_{p} \leq C_{p}\|u\|_{p}
$$

The optimal values of the constants $C_{p}$ were determined by Pichorides [12] and, independently by Cole (unpublished; see Gamelin [8]): we have $C_{p}=$ $\cot \left(\pi /\left(2 p^{*}\right)\right)$ for $1<p<\infty$, where $p^{*}=\max \{p, p /(p-1)\}$. For $p=1$ the

Received January 14, 2011.

2010 Mathematics Subject Classification. Primary 60G44; Secondary 31B05.

Key words and phrases. harmonic function, martingale, LlogL inequality, differential subordination, best constants.

Partially supported by MNiSW Grant N N201 364436.

(C)2012 The Korean Mathematical Society 
inequality (1.1) does not hold with any finite $C_{1}$, but, as shown by Zygmund [17], there are absolute $K, L \geq 0$ such that

$$
\|\tilde{u}\|_{1} \leq K\|u\|_{L \log L}+L,
$$

provided $u$ belongs to the class LlogL. The latter amounts to saying that

$$
\|u\|_{L \log L}=\sup _{0<r<1} \int_{-\pi}^{\pi}\left|u\left(r e^{i \phi}\right)\right| \log ^{+}\left|u\left(r e^{i \phi}\right)\right| \frac{\mathrm{d} \phi}{2 \pi}<\infty .
$$

This has been strenghtened by Pichorides in [12]. He showed that for any $K>2 / \pi$ there is $L<\infty$ depending only on $K$ for which (1.2) is valid, while for $K \leq 2 / \pi$ the inequality fails to hold, no matter how large $L$ we take. A further result in this direction is due to Essén, Shea and Stanton [7]. Using Cole's argument, which transferred the problem of proving a given inequality for conjugate harmonic functions to the problem of constructing an appropriate subharmonic majorant, they proved the existence of absolute constants $C_{1}$, $C_{2}>0$ for which

(1.3) $\int_{-\pi}^{\pi}|\tilde{u}| \leq \frac{2}{\pi} \int_{-\pi}^{\pi}|u| \log (e+|u|)+\frac{4}{\pi} \int_{-\pi}^{\pi}|u| \log \log (e+|u|)+C_{1}|| u \|_{1}+C_{2}$.

Here, as above, the integration is with respect to the normalized Lebesgue's measure. Moreover, they showed that the constant $2 / \pi$ is the best possible, and $4 / \pi$ cannot be replaced by a constant less than $2 / \pi$. See also [6] for a related estimate, in which both constants are equal to $2 / \pi$, but the error term is somewhat different.

In the present paper, we come back to (1.2) and determine the optimal value of the constant $L=L(K)$ for any $K>2 / \pi$. Throughout, $\Phi, \Psi: \mathbb{R} \rightarrow[0, \infty)$ will be given by

$$
\Phi(t)= \begin{cases}|t| & \text { if }|t| \leq 1 \\ \exp (|t|-1) & \text { if }|t|>1\end{cases}
$$

and $\Psi(t)=|t| \log ^{+}|t|$, with the convention $0 \log ^{+} 0=0$. One of our main results can be stated as follows.

Theorem 1.1. For any $K>2 / \pi$, the optimal value $L(K)$ of the constant $L$ in (1.2) is given by

$$
L(K)=K^{2} \int_{0}^{\infty} \frac{\Phi(t)}{\cosh (\pi K t / 2)} d t .
$$

In fact, we shall prove much more and establish a probabilistic counterpart of the theorem above. Let $(\Omega, \mathcal{F}, \mathbb{P})$ be a complete probability space filtered by a nondecreasing family $\left(\mathcal{F}_{t}\right)_{t \geq 0}$ of sub- $\sigma$-algebras of $\mathcal{F}$. In addition, assume that $\mathcal{F}_{0}$ contains all the events of probability 0 . In what follows, $X=\left(X_{t}\right)_{t \geq 0}$, $Y=\left(Y_{t}\right)_{t \geq 0}$ will be two real valued martingales adapted to $\left(\mathcal{F}_{t}\right)_{t \geq 0}$. Let $[X, Y]$ denote the quadratic covariance process between $X$ and $Y$ (for details, see e.g. Dellacherie and Meyer [5]). Following Bañuelos and Wang [1] and Wang [16], we say that $Y$ is differentially subordinate to $X$, if the process 
$\left([X, X]_{t}-[Y, Y]_{t}\right)_{t \geq 0}$ is nondecreasing and nonnegative as a function of $t$. We say that $X$ and $Y$ are orthogonal, if the process $[X, Y]$ is constant. Under the assumption of differential subordination and orthogonality, Bañuelos and Wang [1] proved the following fact, which is a probabilistic version of Riesz's inequality (1.1). We use the notation $\|X\|_{p}=\sup _{t \geq 0}\left\|X_{t}\right\|_{p}$ for $1 \leq p \leq \infty$.

Theorem 1.2. Assume that $X, Y$ are orthogonal martingales such that $Y$ is differentially subordinate to $X$. Then

$$
\|Y\|_{p} \leq \cot \left(\frac{\pi}{2 p^{*}}\right)\|X\|_{p}, \quad 1<p<\infty,
$$

and the inequality is sharp.

This inequality has some further applications to Riesz transforms. See also [2], [3], [9] and [10] for other related results on orthogonal martingales.

Our contribution in this direction is the following version of Theorem 1.1.

Theorem 1.3. Let $X, Y$ be orthogonal martingales such that $Y$ is differentially subordinate to $X$ and $Y_{0}=0$. Then for any $K>2 / \pi$ we have

$$
\|Y\|_{1} \leq \sup _{t \geq 0} \mathbb{E}\left|X_{t}\right| \log ^{+}\left|X_{t}\right|+K^{2} \int_{0}^{\infty} \frac{\Phi(t)}{\cosh (\pi K t / 2)} d t
$$

and the inequality is sharp.

This theorem allows us to obtain the extension of (1.2) to the case when the harmonic functions are defined on Euclidean domains. Suppose that $d$ is a fixed positive integer, $D$ is an open connected subset of $\mathbb{R}^{d}$ and let $u$ and $v$ be real-valued harmonic functions on $D$. Following Burkholder [4], we say that $v$ is differentially subordinate to $u$, if

$$
|\nabla v(x)| \leq|\nabla u(x)| \quad \text { for } x \in D .
$$

The functions $u, v$ are said to be orthogonal if

$$
\nabla u(x) \cdot \nabla v(x)=0 \quad \text { for } x \in D,
$$

where the dot $\cdot$ is the standard scalar product in $\mathbb{R}^{d}$. Note that if $d=2, D$ is a unit disc of $\mathbb{R}^{2}$ and $u, v$ satisfy Cauchy-Riemann equations, then (1.5) and (1.6) hold; thus, these two conditions extend the classical setting of conjugate harmonic functions.

Fix a point $\xi \in D$ and let $D_{0}$ be a bounded connected subdomain of $D$, satisfying $\xi \in D_{0} \subset D_{0} \cup \partial D_{0} \subset D$. Denote by $\mu_{D_{0}}^{\xi}$ the harmonic measure on $\partial D_{0}$ with respect to $\xi$ and let

$$
\|u\|_{p}=\left[\sup _{D_{0}} \int_{\partial D_{0}}|u(x)|^{p} \mathrm{~d} \mu_{D_{0}}^{\xi}(x)\right]^{1 / p}, \quad 1 \leq p<\infty,
$$

and

$$
\|\left. u\right|_{L \log L}=\sup _{D_{0}} \int_{\partial D_{0}}|u(x)| \log ^{+}|u(x)| \mathrm{d} \mu_{D_{0}}^{\xi}(x),
$$


where the supremum is taken over all $D_{0}$ as above.

The generalization of Zygmund's inequality is as follows.

Theorem 1.4. Suppose that $u, v$ are orthogonal harmonic functions on $D, v$ is differentially subordinate to $u$ and $v(\xi)=0$. Then

$$
\|v\|_{1} \leq K\|u\|_{L \log L}+L(K)
$$

and the constant $L(K)$ is the best possible.

A few words are given about the organization of the paper and the method of proof. In the next section, using certain special superharmonic functions, we establish the inequality (1.4) and show how it leads to (1.7). In the proof of (1.4), we base on a duality-type argument and the theory of optimal stopping (see also [10]). Section 3 is devoted to the sharpness of (1.2), which, in turn, implies that the constant $L(K)$ is also the best possible in the preceding estimates (1.4) and (1.7).

\section{Proof of (1.2) and (1.4)}

Suppose that $K>2 / \pi$ is a fixed number. Let $H=\mathbb{R}_{+}^{2}=\mathbb{R} \times[0, \infty)$ denote the upper halfplane and introduce the harmonic function $\mathcal{U}: \mathbb{R} \times(0, \infty) \rightarrow \mathbb{R}$, given by the Poisson integral

$$
\mathcal{U}(\alpha, \beta)=\frac{K}{\pi} \int_{-\infty}^{\infty} \frac{\beta \Phi\left(\left|\frac{2}{K \pi} \log \right| t||\right)}{(\alpha-t)^{2}+\beta^{2}} \mathrm{dt} .
$$

It is easy to check that $\mathcal{U}$ satisfies

$$
\lim _{(\alpha, \beta) \rightarrow(t, 0)} \mathcal{U}(\alpha, \beta)=K \Phi\left(\left|\frac{2}{K \pi} \log \right| t||\right) \quad \text { for } t \neq 0 .
$$

Let $\phi(z)=i e^{-i \pi z / 2}$ be the conformal mapping, which maps the strip $S=$ $[-1,1] \times \mathbb{R}$ onto $H$. Define $U: S \rightarrow \mathbb{R}$ by the formula

$$
U(x, y)= \begin{cases}\mathcal{U}(\phi(x, y)) & \text { if }|x|<1 \\ K \Phi(|y| / K) & \text { if }|x|=1 .\end{cases}
$$

Clearly, $U$ is harmonic in the interior of $S$. By (2.2), it is continuous on this strip.

Lemma 2.1. The function $U$ enjoys the following properties.

(i) $U(x, y)=U(x,-y)=U(-x, y)$ for all $(x, y) \in S$.

(ii) For any $x \in(-1,1)$ and $y \in \mathbb{R}$ we have $U_{y y}(x, y) \geq 0$ and $U_{x x}(x, y) \leq 0$.

(iii) For any $x \in[-1,1]$ we have $U(x, 0) \leq U(0,0)=L(K)$.

(iv) For any $(x, y) \in S, U(x, y) \geq K \Phi(|y| / K)$.

Proof. (i) This is an immediate consequence of the following properties of $\mathcal{U}$ : for all $\alpha \in \mathbb{R}$ and $\beta>0$,

$$
\mathcal{U}(\alpha, \beta)=\mathcal{U}(-\alpha, \beta)=\mathcal{U}\left(\frac{\alpha}{\sqrt{\alpha^{2}+\beta^{2}}}, \frac{\beta}{\sqrt{\alpha^{2}+\beta^{2}}}\right),
$$


which can be established by substituting $t:=-t$ and $t:=1 / t$ in $(2.1)$.

(ii) It suffices to prove the first estimate, then the second follows immediately from the harmonicity of $U$. We have, after the substitution $t=s \exp (\pi y / 2)$,

$$
U(x, y)=\frac{K}{\pi} \int_{-\infty}^{\infty} \frac{\cos \left(\frac{\pi}{2} x\right) \Phi\left(\left|\frac{2}{K \pi} \log \right| s\left|+\frac{y}{K}\right|\right)}{\left(s-\sin \left(\frac{\pi}{2} x\right)\right)^{2}+\cos ^{2}\left(\frac{\pi}{2} x\right)} \mathrm{d} s .
$$

Since for any $s \in \mathbb{R}$, the function $y \mapsto \Phi\left(\left|\frac{2}{K \pi} \log \right| s\left|+\frac{y}{K}\right|\right)$ is convex, the claim follows.

(iii) Since $U_{x x} \leq 0$, (i) implies that $x \mapsto U(x, 0)$ is nonincreasing on $[0,1]$. Thus, for all $x \in[-1,1]$,

$$
\begin{aligned}
U(x, 0) \leq U(0,0)=\mathcal{U}(0,1) & =\frac{2 K}{\pi} \int_{0}^{\infty} \frac{\Phi\left(\left|\frac{2}{K \pi} \log t\right|\right)}{t^{2}+1} \mathrm{dt} \\
& =\frac{4 K}{\pi} \int_{1}^{\infty} \frac{\Phi\left(\frac{2}{K \pi} \log t\right)}{t^{2}+1} \mathrm{~d} t \\
& =K^{2} \int_{0}^{\infty} \frac{\Phi(t)}{\cosh (\pi K t / 2)} \mathrm{d} t .
\end{aligned}
$$

(iv) Fix $y \in \mathbb{R}$ and apply Jensen's inequality in (2.3), with respect to the convex function $t \mapsto K \Phi(|t+y| / K)$. We get

$$
U(x, y) \geq K \Phi\left(\left|\int_{-\infty}^{\infty} \frac{2}{K \pi} \log \right| s\left|\cdot \frac{1}{\pi} \frac{\cos \left(\frac{\pi}{2} x\right)}{\left(s-\sin \left(\frac{\pi}{2} x\right)\right)^{2}+\cos ^{2}\left(\frac{\pi}{2} x\right)} \mathrm{d} s+\frac{y}{K}\right|\right),
$$

which is the claim, since the integral inside is equal to 0 .

In what follows, we will need the following auxiliary fact. Recall that for any real martingale $X$, there exists a unique continuous local martingale part $X^{c}$ of $X$ satisfying

$$
[X, X]_{t}=\left|X_{0}\right|^{2}+\left[X^{c}, X^{c}\right]_{t}+\sum_{0<s \leq t}\left|\triangle X_{s}\right|^{2}
$$

for all $t \geq 0$. Here $\triangle X_{s}=X_{s}-X_{s-}$ denotes the jump of $X$ at time $s$. Furthermore, we have that $\left[X^{c}, X^{c}\right]=[X, X]^{c}$, the pathwise continuous part of $[X, X]$. Here is Lemma 2.1 from $[3]$.

Lemma 2.2. If $X$ and $Y$ are semimartingales, then $Y$ is differentially subordinate and orthogonal to $X$ if and only if $Y^{c}$ is differentially subordinate and orthogonal to $X^{c},\left|Y_{0}\right| \leq\left|X_{0}\right|$ and $Y$ has continuous paths.

Now we are ready to establish the following estimate, which can be regarded as a dual to (1.4).

Theorem 2.3. Suppose that $X$ and $Y$ are orthogonal martingales satisfying the conditions $\|X\|_{\infty} \leq 1, Y_{0} \equiv 0$ and such that $Y$ is differentially subordinate to $X$. Then for any $K>2 / \pi$,

$$
K \sup _{t \geq 0} \mathbb{E} \Phi\left(\left|Y_{t}\right| / K\right) \leq L(K) .
$$


The inequality is sharp.

Proof. Let $t \geq 0$ be fixed. Since $U$ is of class $C^{\infty}$ in the interior of the strip $S$, we may apply Itô's formula to obtain

$$
U\left(X_{t}, Y_{t}\right)=U\left(X_{0}, Y_{0}\right)+I_{1}+I_{2}+\frac{1}{2} I_{3}+I_{4}
$$

where

$$
\begin{aligned}
I_{1} & =\int_{0+}^{t} U_{x}\left(X_{s-}, Y_{s}\right) \mathrm{d} X_{s}+\int_{0+}^{t} U_{y}\left(X_{s-}, Y_{s}\right) \mathrm{d} Y_{s}, \\
I_{2} & =\int_{0+}^{t} U_{x y}\left(X_{s-}, Y_{s}\right) \mathrm{d}\left[X^{c}, Y\right]_{s}, \\
I_{3} & =\int_{0+}^{t} U_{x x}\left(X_{s-}, Y_{s}\right) \mathrm{d}\left[X^{c}, X^{c}\right]_{s}+\int_{0+}^{t} U_{y y}\left(X_{s-}, Y_{s}\right) \mathrm{d}[Y, Y]_{s}, \\
I_{4} & =\sum_{0<s \leq t}\left[U\left(X_{s}, Y_{s}\right)-U\left(X_{s-}, Y_{s}\right)-U_{x}\left(X_{s-}, Y_{s}\right) \Delta X_{s}\right] .
\end{aligned}
$$

Note that we have used above the equalities $Y_{s-}=Y_{s}$ and $Y=Y^{c}$, which are due to the continuity of paths of $Y$. By Lemma 2.1(iii) we have $U\left(X_{0}, Y_{0}\right) \leq$ $L(K)$. The term $I_{1}$ has zero expectation, by the properties of the stochastic integrals. We have $I_{2}=0$ in view of the orthogonality of $X$ and $Y$. The differential subordination together with Lemma 2.1(ii) imply

$$
I_{3} \leq \int_{0}^{t} U_{x x}\left(X_{s}, Y_{s}\right) d\left[X^{c}, X^{c}\right]_{s}+\int_{0}^{t} U_{y y}\left(X_{s}, Y_{s}\right) d\left[X^{c}, X^{c}\right]_{s}=0 .
$$

Finally, we have that $I_{4} \leq 0$, by the concavity of $U(\cdot, y)$ for any fixed $y \in \mathbb{R}$ : see Lemma 2.1(ii). Therefore, by the last part of that lemma,

$$
K \mathbb{E} \Phi\left(\left|Y_{t}\right| / K\right) \leq \mathbb{E} U\left(X_{t}, Y_{t}\right) \leq L(K)
$$

and it suffices to take supremum over $t$ to obtain (2.4). The optimality of the constant $L(K)$ on the right will follow from the sharpness of (1.2): see Remark 3.1 in Section 3 below.

Let us turn to the logarithmic estimate (1.4). It will be studied by means of the following optimal stopping problem. Fix $K>2 / \pi$ and let $B=\left(B^{(1)}, B^{(2)}\right)$ be a two-dimensional Brownian motion starting from $(0,0)$. Introduce the function $V: \mathbb{R}^{2} \rightarrow(-\infty, \infty]$ by

$$
V(x, y)=\sup \mathbb{E} G\left(x+B_{\tau}^{(1)}, y+B_{\tau}^{(2)}\right),
$$

where $G(x, y)=|y|-K|x| \log ^{+}|x|$ and the supremum is taken over all bounded stopping times of $B$. As we shall see, $V$ is the special superharmonic function on which the proof of (1.4) will be based. The interesting fact is that we will not find the explicit formula for $V$; fortunately, we will manage to extract all the required properties directly from the definition (2.7). This is done in the following lemma. 
Lemma 2.4. (i) For any $x \in \mathbb{R}$, we have $V(x, 0) \leq L(K)$.

(ii) The function $V$ is finite on $\mathbb{R}^{2}$.

(iii) The function $V$ is a superharmonic majorant of $G$.

(iv) For any fixed $x \in \mathbb{R}$, the function $V(x, \cdot)$ is convex.

(v) For any $x \in \mathbb{R}$ and $y_{1}, y_{2} \in \mathbb{R}$ we have

$$
\left|V\left(x, y_{1}\right)-V\left(x, y_{2}\right)\right| \leq\left|y_{1}-y_{2}\right| \text {. }
$$

Proof. (i) Fix a bounded stopping time $\tau$ and let $t \geq 0$. We have

$$
\mathbb{E}\left|B_{\tau \wedge t}^{(2)}\right|=\mathbb{E} B_{\tau \wedge t}^{(2)} \operatorname{sgn} B_{\tau \wedge t}^{(2)} .
$$

Consider a martingale $\zeta^{t}=\left(\zeta_{r}^{t}\right)_{r \geq 0}$ given by $\zeta_{r}^{t}=\mathbb{E}\left[\operatorname{sgn} B_{\tau \wedge t}^{(2)} \mid \mathcal{F}_{\tau \wedge r}\right]$. There exists an $\mathbb{R}^{2}$-valued predictable process $A=\left(A_{r}^{(1)}, A_{r}^{(2)}\right)_{r \geq 0}$ such that for all $r$,

$$
\zeta_{r}^{t}=\mathbb{E} \zeta_{t}^{t}+\int_{0+}^{\tau \wedge r} A_{s} d B_{s}=\int_{0+}^{\tau \wedge r} A_{s} d B_{s}
$$

(see e.g. Chapter V in Revuz and Yor [15]). Therefore, using the properties of stochastic integrals, we may write

$$
\begin{aligned}
\mathbb{E}\left|B_{\tau \wedge t}^{(2)}\right| & =\mathbb{E} B_{\tau \wedge t}^{(2)} \int_{0+}^{\tau \wedge t} A_{s} d B_{s} \\
& =\mathbb{E} \int_{0+}^{\tau \wedge t}(0,1) d B_{s} \int_{0}^{\tau \wedge t} A_{s} d B_{s} \\
& =\mathbb{E} \int_{0+}^{\tau \wedge t} A_{s}^{(2)} d s \\
& =\mathbb{E} \int_{0}^{\tau \wedge t}(1,0) d B_{s} \int_{0+}^{\tau \wedge t}\left(A_{s}^{(2)},-A_{s}^{(1)}\right) d B_{s} \\
& =\mathbb{E} B_{\tau \wedge t}^{(1)} \int_{0+}^{\tau \wedge t}\left(A_{s}^{(2)},-A_{s}^{(1)}\right) d B_{s} \\
& =\mathbb{E}\left(x+B_{\tau \wedge t}^{(1)}\right) \int_{0+}^{\tau \wedge t}\left(A_{s}^{(2)},-A_{s}^{(1)}\right) d B_{s} .
\end{aligned}
$$

We easily check that $\Psi$ is the Legendre transform of $\Phi$ and, consequently, for any $a, b \geq 0$ we have

$$
a b \leq \Psi(a)+\Psi(b)
$$

(this inequality can also be verified directly). Apply this to $a=\left|x+B_{\tau \wedge t}^{(1)}\right|$ and $b=\left|\int_{0+}^{\tau \wedge t}\left(A_{s}^{(2)},-A_{s}^{(1)}\right) d B_{s}\right| / K$, and combine the result with the above chain of identities. We obtain

$$
\mathbb{E}\left|B_{\tau \wedge t}^{(2)}\right| \leq K \mathbb{E} \Psi\left(\left|x+B_{\tau \wedge t}^{(1)}\right|\right)+K \Phi\left(\left|\int_{0+}^{\tau \wedge t}\left(A_{s}^{(2)},-A_{s}^{(1)}\right) d B_{s}\right| / K\right) .
$$


Observe that the martingale

$$
\left(\eta_{r}^{t}\right)_{r \geq 0}=\left(\int_{0+}^{\tau \wedge r}\left(A_{s}^{(2)},-A_{s}^{(1)}\right) d B_{s}\right)_{r \geq 0}
$$

is differentially subordinate and orthogonal to $\zeta^{t}$, and starts from 0 . Furthermore, we have $\left\|\zeta^{t}\right\|_{\infty}=\left\|\operatorname{sgn} B_{\tau \wedge t}^{(2)}\right\|_{\infty}=1$, so, by (2.4), we get

$$
\mathbb{E}\left|B_{\tau \wedge t}^{(2)}\right|-K \mathbb{E} \Psi\left(\left|x+B_{\tau \wedge t}^{(1)}\right|\right) \leq L(K) .
$$

This is precisely the desired inequality: it suffices to let $t \rightarrow \infty$ and take supremum over $\tau$.

(ii) Take a bounded stopping time $\tau$ and note that the process $\left(B_{\tau \wedge t}^{(2)}\right)_{t \geq 0}$ is differentially subordinate and orthogonal to $\left(x+B_{\tau \wedge t}^{(1)}\right)_{t \geq 0}$. Therefore, by (2.8), for any $t \geq 0$,

$$
\begin{aligned}
\mathbb{E}\left|y+B_{\tau \wedge t}^{(2)}\right|-K \mathbb{E} \Psi\left(\left|x+B_{\tau \wedge t}^{(1)}\right|\right) & \leq|y|+\mathbb{E}\left|B_{\tau \wedge t}^{(2)}\right|-K \mathbb{E} \Psi\left(\left|x+B_{\tau \wedge t}^{(1)}\right|\right) \\
& \leq|y|+L(K)
\end{aligned}
$$

and this gives the finiteness of $V$, since $\tau$ was arbitrary.

(iii) To show the majorization $V \geq G$, it suffices to consider in (2.7) the stopping time $\tau \equiv 0$. The superharmonicity can be established using standard Markovian arguments (see e.g. Chapter I in [11]).

(iv) Fix $x, y_{1}, y_{2} \in \mathbb{R}$ and $\lambda \in(0,1)$. For any bounded $\tau$, we have, using the triangle inequality,

$$
\begin{aligned}
\mathbb{E} G\left(x+B_{\tau}^{(1)}, \lambda y_{1}+(1-\lambda) y_{2}+B_{\tau}^{(2)}\right) \leq & \lambda \mathbb{E} G\left(x+B_{\tau}^{(1)}, y_{1}+B_{\tau}^{(2)}\right) \\
& +(1-\lambda) \mathbb{E} G\left(x+B_{\tau}^{(1)}, y_{2}+B_{\tau}^{(2)}\right) \\
\leq & \lambda V\left(x, y_{1}\right)+(1-\lambda) V\left(x, y_{2}\right) .
\end{aligned}
$$

It remains to take supremum over $\tau$ to get the claim.

(v) The reasoning is similar to that from the previous part. Let $\tau$ be a bounded stopping time. We have

$$
G\left(x+B_{\tau}^{(1)}, y_{1}+B_{\tau}^{(2)}\right) \leq\left|y_{1}-y_{2}\right|+G\left(x+B_{\tau}^{(1)}, y_{2}+B_{\tau}^{(2)}\right),
$$

so integrating and taking supremum over $\tau$ gives $V\left(x, y_{1}\right)-V\left(x, y_{2}\right) \leq\left|y_{1}-y_{2}\right|$. The claim follows from the symmetry of the roles of $y_{1}$ and $y_{2}$.

Now we turn to the martingale version of Zygmund's inequality.

Proof of (1.4). We may and do assume that

$$
\sup _{t \geq 0} \mathbb{E}\left|X_{t}\right| \log ^{+}\left|X_{t}\right|<\infty,
$$

since otherwise there is nothing to prove. Obviously, we will be done if we show that for any $t \geq 0$,

$$
\mathbb{E}\left|Y_{t}\right| \leq K \mathbb{E}\left|X_{t}\right| \log ^{+}\left|X_{t}\right|+L(K)
$$


We shall proceed as in the proof of (2.4), with $U$ replaced by $V$. However, we need some extra effort, since $V$ need not be of class $C^{2}$ and we cannot apply Itô's formula directly. To overcome this difficulty, we make use of the following approximation argument. Fix $\delta \in(0,1)$ and consider a $C^{\infty}$ function $g: \mathbb{R}^{2} \rightarrow[0, \infty)$, supported on the ball with center $(0,0)$ and radius $\delta$, satisfying $\left\|g^{\delta}\right\|_{1}=1$. Introduce $G^{\delta}, V^{\delta}$ by the convolutions

$$
\begin{aligned}
G^{\delta}(x, y) & =\int_{\mathbb{R}^{2}} G(x-a \delta, y-b \delta) g(a, b) \mathrm{d} a \mathrm{~d} b, \\
V^{\delta}(x, y) & =\int_{\mathbb{R}^{2}} V(x-a \delta, y-b \delta) g(a, b) \mathrm{d} a \mathrm{~d} b,
\end{aligned}
$$

These two functions are of class $C^{\infty}$ and inherit the key properties from $G$ and $V$. To be more specific, we have the majorization $G^{\delta} \leq V^{\delta}$, the function $V^{\delta}$ is superharmonic and we have $V_{y y}^{\delta} \geq 0$; thus, $V_{x x}^{\delta} \leq 0$ on $\mathbb{R}^{2}$. Now we are allowed to apply Itô's formula, which yields

$$
V^{\delta}\left(X_{t}, Y_{t}\right)=V^{\delta}\left(X_{0}, Y_{0}\right)+I_{1}+\frac{1}{2} I_{2}+\frac{1}{2} I_{3}+I_{4}
$$

where $I_{1}, I_{2}, I_{3}$ and $I_{4}$ are analogous to the terms in (2.5) (simply replace $U$ by $V^{\delta}$ there). If we repeat the reasoning from the proof of (2.4), we get that $\mathbb{E} I_{1}=0$ and $I_{2}, I_{3}, I_{4}$ are nonpositive. In consequence, we have

$$
\mathbb{E} G^{\delta}\left(X_{t}, Y_{t}\right) \leq \mathbb{E} V^{\delta}\left(X_{t}, Y_{t}\right) \leq \mathbb{E} V^{\delta}\left(X_{0}, Y_{0}\right)=\mathbb{E} V^{\delta}\left(X_{0}, 0\right) .
$$

Now we are going to let $\delta \rightarrow 0$. To see what happens, note that using parts (i) and $(\mathrm{v})$ of Lemma 2.4, we get

$$
V^{\delta}(x, 0) \leq \int_{\mathbb{R}^{2}}[V(x-a \delta, 0)+b \delta] g(a, b) \mathrm{d} a \mathrm{~d} b \leq L(K)+\delta .
$$

Similar reasoning yields

$$
\begin{aligned}
G^{\delta}(x, y) & \geq \int_{\mathbb{R}^{2}}\left[|y|-b \delta-K|x-a \delta| \log ^{+}|x-a \delta|\right] g(a, b) \mathrm{d} a \mathrm{~d} b \\
& \geq|y|-\delta-K \int_{\mathbb{R}^{2}}|x-a \delta| \log ^{+}|x-a \delta| g(a, b) \mathrm{d} a \mathrm{~d} b .
\end{aligned}
$$

Consequently, we obtain

$$
\mathbb{E}\left|Y_{t}\right| \leq K \mathbb{E} \int_{\mathbb{R}^{2}}\left|X_{t}-a \delta\right| \log ^{+}\left|X_{t}-a \delta\right| g(a, b) \mathrm{d} a \mathrm{~d} b+L(K)+2 \delta .
$$

Since for some absolute $c_{1}, c_{2}$ we have

$$
|x-\delta| \log ^{+}|x-\delta| \leq c_{1}|x| \log ^{+}|x|+c_{2} \quad \text { for } x \in \mathbb{R}, \delta \in(0,1),
$$

it remains to use Lebesgue's dominated convergence theorem and (2.9) to obtain (2.10). 
Proof of (1.7). Fix a bounded subdomain $D_{0}$ of $D$ satisfying $\xi \in D_{0} \subset D_{0} \cup$ $\partial D_{0} \subset D$. Let $B=\left(B_{t}\right)_{t \geq 0}$ be an $d$-dimensional Brownian motion starting from $\xi$ and let $\tau$ denote the first moment $B$ hits the boundary of $D_{0}$. Consider martingales $X, Y$ given by $X_{t}=u\left(B_{\tau \wedge t}\right)$ and $Y_{t}=v\left(B_{\tau \wedge t}\right)$ for $t \geq 0$. We have

$$
\begin{aligned}
{[X, X]_{t} } & =u^{2}(\xi)+\int_{0}^{\tau \wedge t}\left|\nabla u\left(B_{s}\right)\right|^{2} \mathrm{~d} s, \\
{[Y, Y]_{t} } & =v^{2}(\xi)+\int_{0}^{\tau \wedge t}\left|\nabla v\left(B_{s}\right)\right|^{2} \mathrm{~d} s, \\
{[X, Y]_{t} } & =u(\xi) v(\xi)+\int_{0}^{\tau \wedge t} \nabla u\left(B_{s}\right) \cdot \nabla v\left(B_{s}\right) \mathrm{d} s
\end{aligned}
$$

and we see that the assumptions on $u$ and $v$ imply that $Y_{0} \equiv 0, Y$ is differentially subordinate to $X$ and that $X, Y$ are orthogonal. Therefore, by (1.4),

$$
\begin{aligned}
\int_{\partial D_{0}}|v(x)| d \mu_{D_{0}}^{\xi}(x) & =\|Y\|_{1} \leq K \sup _{t \geq 0} \mathbb{E}\left|X_{t}\right| \log ^{+}\left|X_{t}\right|+L(K) \\
& =K \int_{\partial D_{0}}|u(x)| \log ^{+}|u(x)| \mathrm{d} \mu_{D_{0}}^{\xi}(x)+L(K) .
\end{aligned}
$$

It suffices to take supremum over all $D_{0}$ to complete the proof.

As we have already mentioned above, the choice $d=2, D=\{(x, y)$ : $\left.x^{2}+y^{2} \leq 1\right\}$ and $\xi=(0,0)$ leads to Zygmund's inequality (1.2) for conjugate harmonic functions.

\section{Sharpness}

Clearly, it suffices to prove that the inequality (1.2) is sharp. For each $K>2 / \pi$, we will exhibit a function $u$ for which its (normalized) conjugate $\tilde{u}$ satisfies

$$
\|\tilde{u}\|_{1} \geq K\|u\|_{L \log L}+L(K) .
$$

Recall that $S$ denotes the strip $[-1,1] \times \mathbb{R}$. We start with the observation that the mapping $G: \mathbb{D} \rightarrow S$, given by $G(z)=(2 / \pi) \log [(i z-1) /(z-i)]-i$, is conformal and satisfies $G(0)=0$. We easily derive that $w=\operatorname{Re} G$ and its conjugate $\tilde{w}=\operatorname{Im} G$ have the following behavior at $\partial \mathbb{D}$ :

$$
w\left(e^{i \phi}\right)=\frac{2}{\pi} \log \left|\frac{1+\sin \phi}{\cos \phi}\right|, \quad \tilde{w}\left(e^{i \phi}\right)=1_{\{|\phi| \leq \pi / 2\}}-1_{\{|\phi|>\pi / 2\}}, \quad|\phi| \leq \pi .
$$

Introduce the function $u$ on the unit circle by the formula

$$
u\left(e^{i \phi}\right)=\Phi^{\prime}\left(\left|\frac{2}{K \pi} \log \right| \frac{1+\sin \phi}{\cos \phi}||\right) \operatorname{sgn}\left(\log \left|\frac{1+\sin \phi}{\cos \phi}\right|\right), \quad|\phi| \leq \pi,
$$


and extend it to the whole disc $\mathbb{D}$ by the Poisson integral. Recall $\Psi$ defined in the introduction. We have that

$$
\begin{aligned}
\|u\|_{L \log L} & =\frac{1}{2 \pi} \int_{-\pi}^{\pi} \Psi\left(\Phi^{\prime}\left(\left|\frac{2}{K \pi} \log \right| \frac{1+\sin \phi}{\cos \phi}||\right)\right) \mathrm{d} \phi \\
& =\frac{2}{\pi} \int_{0}^{\pi / 2} \Psi\left(\Phi^{\prime}\left(\frac{2}{K \pi} \log \frac{1+\sin \phi}{\cos \phi}\right)\right) \mathrm{d} \phi,
\end{aligned}
$$

which, after substitution $t=\frac{2}{\pi} \log \left(\frac{1+\sin \phi}{\cos \phi}\right)$, becomes

$$
\|u\|_{L \log L}=\int_{0}^{\infty} \frac{\Psi\left(\Phi^{\prime}(t / K)\right)}{\cosh (\pi t / 2)} \mathrm{d} t
$$

On the other hand, since $\|\tilde{w}\|_{\infty}=1$, we have

$$
\begin{aligned}
\|\tilde{u}\|_{1} & \geq \sup _{0<r<1} \int_{-\pi}^{\pi} \tilde{u}\left(r e^{i \phi}\right) \tilde{w}\left(r e^{i \phi}\right) \frac{d \phi}{2 \pi}=\int_{-\pi}^{\pi} u\left(e^{i \phi}\right) w\left(e^{i \phi}\right) \frac{d \phi}{2 \pi} \\
& =\int_{-\pi}^{\pi} \Phi^{\prime}\left(\left|\frac{2}{K \pi} \log \right| \frac{1+\sin \phi}{\cos \phi}||\right)\left|\frac{2}{\pi} \log \right| \frac{1+\sin \phi}{\cos \phi} \| \frac{d \phi}{2 \pi} \\
& =\int_{0}^{\infty} \frac{\Phi^{\prime}(t / K) t}{\cosh (\pi t / 2)} \mathrm{d} t .
\end{aligned}
$$

It suffices to use the identities

$$
\Phi^{\prime}(t) t=\Psi\left(\Phi^{\prime}(t)\right)+\Phi(t), \quad t \geq 0
$$

and

$$
K \int_{0}^{\infty} \frac{\Phi(t / K)}{\cosh (\pi t / 2)} \mathrm{d} t=L(K)
$$

to complete the proof of $(3.1)$.

Remark 3.1. This example shows that the inequality (2.4) is also sharp. Indeed, if it were possible to replace $L(K)$ by a smaller constant there, then the same constant would suffice in (2.8), (2.10) and hence also in (1.2).

\section{References}

[1] R. Banuelos and G. Wang, Sharp inequalities for martingales with applications to the Beurling-Ahlfors and Riesz transforms, Duke Math. J. 80 (1995), no. 3, 575-600.

[2] _ Orthogonal martingales under differential subordination and application to Riesz transforms, Illinois J. Math. 40 (1996), no. 4, 678-691.

[3] _ Davis's inequality for orthogonal martingales under differential subordination, Michigan Math. J. 47 (2000), no. 1, 109-124.

[4] D. L. Burkholder, Differential subordination of harmonic functions and martingales, Harmonic analysis and partial differential equations (El Escorial, 1987), 1-23, Lecture Notes in Math., 1384, Springer, Berlin, 1989.

[5] C. Dellacherie and P. A. Meyer, Probabilities and Potential B, North-Holland, Amsterdam, 1982.

[6] M. Essén, D. F. Shea, and C. S. Stanton, Best constants in Zygmund's inequality for conjugate functions, Papers on analysis, 73-80, Rep. Univ. Jyvaskyla Dep. Math. Stat., 83, University of Jyväskila, 2001. 
[7] _ Sharp L $\log ^{\alpha} L$ inequalities for conjugate functions, Ann. Inst. Fourier (Grenoble) 52 (2002), no. 2, 623-659.

[8] T. W. Gamelin, Uniform Algebras and Jensen Measures, London Math. Soc. Lecture Notes Series, Vol. 32, Cambridge Univ. Press, Cambridge/New York, 1978.

[9] P. Janakiraman, Best weak-type $(p, p)$ constants, $1<p<2$, for orthogonal harmonic functions and martingales, Illinois J. Math. 48 (2004), no. 3, 909-921.

[10] A. Osȩkowski, Sharp inequalities for differentially subordinate harmonic functions and martingales, to appear in Canadian Math. Bull.

[11] G. Peskir and A. Shiryaev, Optimal Stopping and Free Boundary Problems, Lectures in Mathematics ETH Zurich. Birkhäuser Verlag, Basel, 2006.

[12] S. K. Pichorides, On the best values of the constants in the theorems of M. Riesz, Zygmund and Kolmogorov, Studia Math. 44 (1972), 165-179.

[13] M. Riesz, Les fonctions conjugées et ler séries de Fourier, C. R. Acad. Paris, 178 (1924), 1464-1467.

[14] Sur les fonctions conjugées, Math. Z. 27 (1927), 218-244.

[15] D. Revuz and M. Yor, Continuous Martingales and Brownian Motion, 3rd edition, Springer Verlag, 1999.

[16] G. Wang, Differential subordination and strong differential subordination for continuous time martingales and related sharp inequalities, Ann. Probab. 23 (1995), no. 2, 522-551.

[17] A. Zygmund, Sur les fonctions conjugées, Fund. Math. 13 (1929), 284-303.

Department of Mathematics, Informatics and Mechanics

UNIVERSITY OF WARSAW

BANACHA 2, 02-097 WARSAW, POLAND

E-mail address: ados@mimuw.edu.pl 